\title{
MULTIPLE SCLEROSIS DIAGNOSIS WITH FUZZY C-MEANS
}

\author{
Saba Heidari Gheshlaghi ${ }^{1}$, Abolfazl Madani ${ }^{2}$, AmirAbolfazl Suratgar $^{3}$ and \\ Fardin Faraji ${ }^{4}$ \\ ${ }^{1,3}$ Department of Electrical Engineering, Amirkabir University of Technology \\ (Tehran Polytechnic), Tehran, Iran \\ ${ }^{2}$ Department of Control Engineering, South Tehran Branch Islamic Azad \\ University (IAU) Tehran, Iran \\ ${ }^{4}$ Neurology Department, Arak University of Medical Sciences, Arak, Iran
}

\begin{abstract}
Magnetic resonance imaging (MRI) can support and substitute clinical information in the diagnosis of multiple sclerosis (MS) by presenting lesion. In this paper, we present an algorithm for MS lesion segmentation. We revisit the modification of properties of fuzzy $c$ means algorithms and the canny edge detection. Using reformulated fuzzy c means algorithms, apply canny contraction principle, and establish a relationship between MS lesions and edge detection. For the special case of FCM, we derive a sufficient condition for fixed lesions, allowing identification of them as (local) minima of the objective function.
\end{abstract}

\section{KEYWORDS}

Multiple Sclerosis, MRI, T2, fuzzy c-means (FCM), Canny.

\section{INTRODUCTION}

Over time, the importance of correct diagnosing for diseases are increased and it is an essential to find an optimal method for diagnosing disease. Multiple sclerosis, also known as MS, is a chronic disease that attacks the central nervous system(CNS) and affects white matter by the person's own immune system so that MS is known as an auto-immune disease. As we know, Nerve fiber is surrounded by myelin, which protects the nerve and helps it to conduct electrical Signals (Impulse). In MS patients, the myelin is disappeared. Because of this, the MS patients could not move their body properly. MS is a largely unknown disease these days and diagnosis it correctly and early, has a significant impact on disease progression [1]

Brain lesions detection plays an important role in MS studies, as it is used to evaluate patient disease and its future evolution. Currently, lesions are detected manually or semi-automatic segmentation methods, which are very time to consuming and show a high inter and intra-raters variability [2]

Natarajan Meghanathan et al. (Eds) : NLP, JSE, CST, SIP, ARIA - 2018

pp. 97-104, 2018. @ CS \& IT-CSCP 2018

DOI : $10.5121 /$ csit.2018.80210 
Magnetic resonance imaging (MRI) were officially included in the diagnostic workup of patients presenting with a clinically isolated syndrome suggestive of multiple sclerosis in 2001 by an international panel of experts [3]. Diagnosing multiple sclerosis depends on evidence of disease dissemination in space and time and exclusion of other syndromes that can imitate multiple sclerosis by their clinical and laboratory Specifications. MRI can support and substitute clinical information for multiple sclerosis diagnoses, allowing an early and accurate diagnosis. [4] The clinical presentation of MS includes a wide range of physical disorders and cognitive symptoms. Cognitive Impairment decrease quality of life and treatment amongst MS patients [5-8]. MS is an inflammatory demyelinating and degenerative disease of the CNS, distinctive pathologically by a different part of brain inflammation, demyelination, axonal loss, often causing motor, sensorial, vision, correlation, and cognitive impairment [9].

Also, MS is the recurring, neurological disease capable of causing disability in young adults. In these days, getting MS disease has been increased, and geographical areas play an important role in getting MS. Moreover, Multiple sclerosis is between two and three-times more average in females than in males, but males have a propensity for later disease [10]. As mentioned, one of the most famous facilities for diagnosis MS disease is Magnetic Resonance Imaging (MRI) techniques [9], T2-weighted (T2-w) and gadolinium-enhanced T1-weighted (T1-w), are highly aware in detecting MS plaques. MRI-derived metrics have become the most important medical tool for diagnosing MS disease.

Both sharp and persistent MS plaques appear as focal high-signal intensity areas on T2-w sequences, illustrate their increased tissue water content. The total T2 lesion volume of the brain increases by almost (5-10\%) each year in the relapsing forms of MS [11]. Gadolinium-enhanced T1-w imaging is highly fragile in detecting inflammatory activity. CNS atrophy, which involves both gray matter and white matter, is a progressive phenomenon that becomes worse with argument disease length and progresses at a rate of between $0.6 \%$ and $1.2 \%$ of brain loss per year in this disease [12]. It should consider that different method of segmentation might have an important impression on the small volume of the lesion detection.

In this paper, Section 2 reviews of the pre-processing steps needed when automatically segmenting MS lesions. Section 3 approaches segmentation methods and also reviews algorithms which are used. In Section 4, conclusion and results are showing and give some ideas for future works.

In this research, MRIs for patients with MS are from different sources. Mostly, our high gratitude should be directed to C.P. Loizoua and his colleagues: The Laboratory of Health at the University of Cyprus (http://www.medinfo.cs.ucy.ac.cy/)

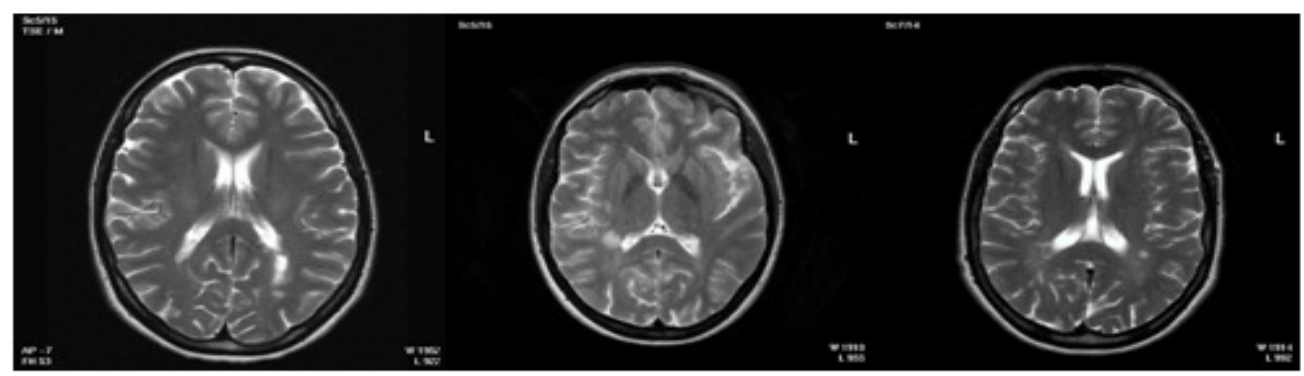

Figure 1: Samples of MR images for MS patient 


\section{Pre-ProceSSING STEPS}

Accurate identification of brain in MRI images is a critical first step in many neuroimaging studies. There are many technics for skull extraction [19, 20]. Although this step is a significant part, using an efficient method is important. In our research, we used the automated brain extraction tool (BET) (Smith, 2002) [13-15]. Also, image binarization is done in this step Eq. (1). th is described as a threshold.

$$
\operatorname{Bin}(x, y)=\left\{\begin{array}{lllll}
1 & & \text { if } & & f(x, y)>t h \\
& 0 & \text { if } & O . w
\end{array}\right\}
$$

\section{SEGMENTATION METHODS AND ALGORITHM}

Varieties of methods for automatic analysis and segmentation have been developed that the image can be segmented into its basic elements.

Edge detection is an image processing technique for finding the boundaries of objects by detecting brightness discontinuities; it is also used for image segmentation and data extraction methods such as image processing, computer vision, and machine vision.

There are various classification methods for edge detection, but they are usually divided into two main groups:

a) Gradient method: The gradient method looks for maximum and minimum in the first derivative of the image for detecting the edges [16].

b) Laplacian method: It searches for zero crossings in the second derivative of the image to find edges. An edge has the one-dimensional shape of a ramp and calculating the derivative of the image can highlight its location. The zero-crossing of the differentiated signal as edge points [16]. The two-dimensional Gaussian operator $\mathrm{G}(\mathrm{x}, \mathrm{y})$ is given by:

$$
G(x, y)=\frac{1}{2 \pi \sigma_{x} \sigma_{y}} e^{-\left(\frac{x^{2}}{2 \sigma_{x}^{2}}+\frac{y^{2}}{2 \sigma_{y}^{2}}\right)}
$$

With condition; i.e. $\sigma_{x}=\sigma_{y}$ and $x^{2}+y^{2}=r^{2}$ the equation simplify to:

$$
G(r)=\frac{1}{2 \pi \sigma^{2}} e^{\frac{r^{2}}{2 \sigma^{2}}}
$$

For detecting edges, searching for zero-crossing in 2nd order derivative of the image needed which $f(x, y)$ is the image [2].

$$
g(x, y)=\nabla^{2} G(r) * f(x, y)
$$




$$
\nabla^{2} G(r)=\frac{-1}{4 \pi \sigma^{2}}\left[1-\frac{r^{2}}{2 \sigma}\right] e^{\frac{r^{2}}{2 \sigma^{2}}}
$$

Common edge detection algorithms consist Sobel, Canny, Prewitt, Marr-Hildreth. Those methods are determined in this research. The main difference between these methods is that canny edge detection method is in the spatial domain while others are in the frequency domain [9].

Below, the differences between Canny, Sobel, and Marr-Hildreth edge detection is shown in figure2. As shown in figure 2, canny edge detection has a better result in comparison with other methods.
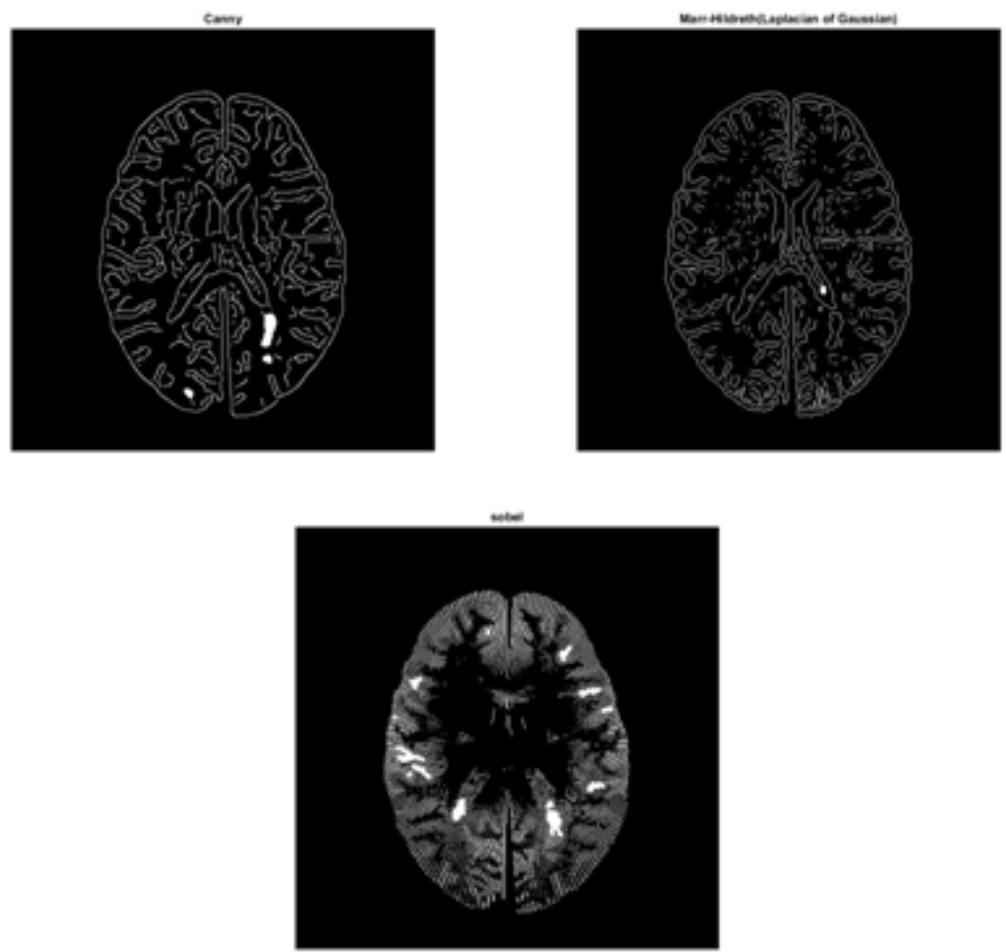

Figure 2: differences between Canny, Sobel and Marr-Hildreth edge detection

\section{FUZZY C-MEANS}

Clustering of numerical data forms of many classification and system modeling methods. The suggestion of clustering is to identify natural groupings of data from a large dataset to create a concise representation of a system's behavior. Fuzzy c-means (FCM) is a data clustering algorithm in which a data set is grouped into $\mathrm{n}$ clusters with any data point in the data set belonging to every cluster to the main degrees [17]. The FCM algorithm assumes that each point belongs to more than one cluster with given a dataset $X=\left\{x_{1}, x_{2}, x_{3} \ldots, x_{N}\right\}$ clustering goals to discrete the dataset into varying subsets each with a cluster midpoint. FCM algorithm apportions fuzzy memberships $u_{i j}$ any pixels $x_{j} \quad(j=1,2, \ldots, N)$ in each variety c by decreasing the following cost function: 


$$
J=\sum_{j=1}^{N} \sum_{i=1}^{C} u_{i j}^{m}\left\|x_{j}-v_{i}\right\|^{2}
$$

Where $\mathrm{m}$ is the weighting fuzziness parameter. $v_{i}$ Illustrate the $\mathrm{i}$-th cluster midpoint. II. I Explain the Euclidean interval. $u_{i j}$ Means a partition matrix that is subject $u_{i j} \in[0,1]$ and $\sum_{i=1}^{c} u_{i j}=1$. Using the Lagrangian method, partition matrix, and cluster centers by calculating as follows:

$$
\begin{gathered}
v_{i}=\frac{\sum_{j=1}^{N} u_{i j}^{m} x_{j}}{\sum_{j=1}^{N} u_{i j}^{m}} \\
u_{i j}=\frac{1}{\sum_{k=1}^{c}\left(\frac{\left\|x_{j}-v_{i}\right\|^{2}}{\left\|x_{j}-v_{k}\right\|^{2}}\right)^{\frac{1}{(m-1)}}}
\end{gathered}
$$

In this study, considering the MR image is often with spatial inhomogeneity due to the defect in the magnetic field, the intensity $x_{j}$ is modeled by $x_{j}=y_{i}-\gamma_{j}$ here, $y_{i}$ and $\gamma_{j}$, and mean the measured absorption and the consistent bias field. We propose a novel algorithm as an addition of the traditional FCM clustering by modifying the cost function in Eq. (9) as [18, 19]:

$$
J=\sum_{j=1}^{N} \sum_{i=1}^{c} u_{i j}^{m}\left\|x_{j}-v_{i}\right\|^{2}+\alpha S+\beta R
$$

Where $\mathrm{S}$ is a normalizer indicating the neighborhood influence during segmentation. $\mathrm{R}$ is the regularization term on bias field. $\alpha$ and $\beta$ are constants that control the effect of the two regularization term correspondingly definitely, S, R are established as [14]:

$$
\begin{aligned}
& S=\sum_{j=1}^{N} \sum_{i=1}^{C} u_{i j}^{m}\left(\frac{1}{N_{\varepsilon}} \sum_{x \in N_{\varepsilon}}\left\|x-v_{i}\right\|^{2}\right) \\
& \text { and } \\
& R=\sum_{j=1}^{N} \sum_{i=1}^{C} u_{i j}^{m}\left(\left\|\gamma_{j}\right\|^{2}\right)
\end{aligned}
$$

Where $N_{\varepsilon}$ represent neighborhood positioning at $x_{j}$. 
Also to the traditional FCM algorithm, by differentiating the cost function respect to, $V_{i}$ and $\gamma_{j}$ respectively and setting the result to zero, we can obtain the updating $u_{i j}^{*}, v_{i}^{*}$ and $\gamma_{j}^{*}$ as:

$$
\begin{aligned}
& u_{i j}^{*}=\frac{1}{\sum_{k=1}^{c}\left(\frac{\left\|x_{j}-v_{i}\right\|^{2}+\alpha \cdot \frac{1}{N_{\varepsilon}} \sum_{x \in N_{\varepsilon}}\left\|x-v_{i}\right\|^{2}+\beta \cdot \gamma_{j}}{\left\|x_{j}-v_{k}\right\|^{2}+\alpha \cdot \frac{1}{N_{\varepsilon}} \sum_{x \in N_{\varepsilon}}\left\|x-v_{k}\right\|^{2}+\beta \cdot \gamma_{j}}\right)^{\frac{1}{(m-1)}}} \\
& v_{i}^{*}=\frac{\sum_{j=1}^{N} u_{i j}^{m}\left(x_{j}+\alpha \cdot \frac{1}{N_{\varepsilon}} \sum_{x \in N_{\varepsilon}}\left(x-v_{i}\right)\right.}{(1+\alpha) \sum_{j=1}^{N} u_{i j}^{m}} \\
& \gamma_{j}^{*}=y_{j}-\frac{(1+\beta) \sum_{i=1}^{c} u_{i j}^{m} v_{i}^{m}}{\left(1+\beta i_{i j}\right.}
\end{aligned}
$$

The result is shown below (fig4). For achieving this result, first, we use the pre-processing method as described in section 2, then applying canny edge detection and finally, for increasing accuracy, FCM is applied.

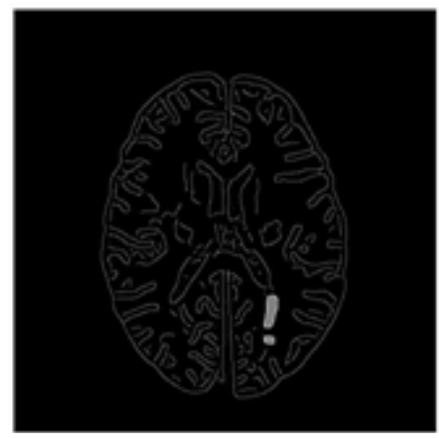

Figure 3: Result of adding FCM after canny edge detection

\section{CONCLUSIONS}

Multiple sclerosis caused when the immune system attacks myelin, which covered around human nerve fibers to protect them and help them to send the messages efficiently.[1] Without this shell, nerves became damaged. Scar tissue may form MS, is a long-lasting disease that can affect the brain, spinal cord, and the optic nerves in eyes. By this damage, transferring messages between brain and body will be affected. The message will slow down or blocked during transferring and 
leading to the symptoms of MS. Doctors use medical history, physical exam, neurological exam, especially MRI to diagnose it. MR images still is a valuable tool for identification multiple sclerosis. There is no cure for MS, but medicines may slow it down and help control the disease progress.[7] Physical and occupational therapy may also be helpful. Because of the sensitivity of MR images, determine the disease clinically is hard and take a long time so having an automatic method for diagnosing the disease fast and accurate is critical.

In our research, different image processing methods are used, such as edge detection and segmentation. Also, we use some famous pre-processing techniques for having the best result, like Brain extraction tool [13-15] and binarization. In addition, we presented a modification method with Fuzzy C Means for better lesion segmentation and using canny for better edge detection. also show that, canny edge detection has a better result. In our method, we first used edge detection method for finding edges and then applying FCM for increasing diagnostic accuracy. In addition, we change clustering parameters to get different results for better lesion detection, Also as it was mentioned before, in MS lesion detection, effectiveness is the most important part. In this paper, lesion diagnosis accuracy is improved in comparison with others works [20].

\section{REFERENCES}

[1] Van Waesberghe, J. H. T. M., et al. "Axonal loss in multiple sclerosis lesions: magnetic resonance imaging insights into substrates of disability." Annals of neurology 46.5 (1999): 747-754.

[2] Beaumont, J., Commowick, O., \& Barillot, C. Multiple Sclerosis lesion segmentation using an automated multimodal Graph Cut. In Proceedings of the 1st MICCAI Challenge on Multiple Sclerosis Lesions Segmentation Challenge Using a Data Management and Processing Infrastructure-MICCAIMSSEG (2016, October): 1-8.

[3] Golkov, Vladimir, Tim Sprenger, Jonathan Sperl, Marion Menzel, Michael Czisch, Philipp Samann, and Daniel Cremers. "Model-free novelty-based diffusion MRI." In Biomedical Imaging (ISBI), 2016 IEEE 13th International Symposium on , (2016) :1233-1236.

[4] Filippi, Massimo, et al. "MRI criteria for the diagnosis of multiple sclerosis: MAGNIMS consensus guidelines." The Lancet Neurology 15.3 (2016): 292-303

[5] Uher, T., et al. "Identification of multiple sclerosis patients at highest risk of cognitive impairment using an integrated brain magnetic resonance imaging assessment approach." European journal of neurology 24.2 (2017): 292-301.

[6] Kjølhede, Tue, et al. "Can resistance training impact MRI outcomes in relapsing-remitting multiple sclerosis?." Multiple Sclerosis Journal (2017): 1-10.

[7] Preziosa, Paolo, et al. "Comparison of MRI Criteria for the Diagnosis of Multiple Sclerosis: Role of Cortical Lesions (S45. 003)." Neurology 86.16 Supplement (2016): S45-003.

[8] Favaretto, Alice, et al. "MRI-detectable cortical lesions in the cerebellum and their clinical relevance in multiple sclerosis." Multiple Sclerosis Journal 22.4 (2016): 494-501.

[9] Denitto, Matteo, et al. "A biclustering approach based on factor graphs and max-sum algorithm." Pattern Recognition 62(2017): 114-124 
[10] A. Compston, A. Coles, Multiple sclerosis, Lancet 359 (9313) (2006) 1221-1231.

[11] S. Saha, S. Bandyopadhyay, A new point symmetry based fuzzy genetic clustering technique for automatic evolution of clusters, Inf. Sci. 179 (9) (2009): 3230-3246.

[12] H. McFarland, L. Stone, P. Calabresi, H. Maloni, C. Bash, J. Frank, MRI studies of multiple sclerosis: implications for the natural history of the disease and for monitoring effectiveness of experimental therapies, Mult. Scler. 2 (4) (1996): 198-205.

[13] Popescu, Valeriu, et al. "Optimizing parameter choice for FSL-Brain Extraction Tool (BET) on 3D T1 images in multiple sclerosis." NeuroImage 61.4 (2012): 1484-1494.

[14] Eskildsen, Simon F., et al. "BEaST: brain extraction based on nonlocal segmentation technique." NeuroImage 59.3 (2012): 2362-2373.

[15] Rex, David E., et al. "A meta-algorithm for brain extraction in MRI." NeuroImage 23.2 (2004): 625637.

[16] Walker, Stephen G. "A Laplace transform inversion method for probability distribution functions." Statistics and Computing 27.2 (2017): 439-448.

[17] Meena Prakash, R., and R. Shantha Selva Kumari. "Fuzzy C means integrated with spatial information and contrast enhancement for segmentation of MR brain images." International Journal of Imaging Systems and Technology 26.2 (2016): 116-123.

[18] Horsfield, Mark A., et al. "Rapid semi-automatic segmentation of the spinal cord from magnetic resonance images: application in multiple sclerosis." Neuroimage 50.2 (2010): 446-455.

[19] L.P. Clarke, R.P. Velthuizen, M.A. Camacho, J.J. Heine, M. Vaidyanathan, L.O. Hall, R.W. Thatcher, M.L. Silbiger, MRI segmentation: methods and applications, Magn. Reson. Imag. 13 (3) (1995): 343368.

[20] Ali, S. M., and Asmaa Maher. "Identifying multiple sclerosis lesions in MR images using image processing techniques." Multidisciplinary in IT and Communication Science and Applications (AICMITCSA), Al-Sadeq International Conference on. IEEE, (2016):1-4 